\title{
Um algoritmo de Membership para o modelo Síncrono Particionado (SPA) de Sistemas Distribuídos com particionamento forte.
}

\author{
Maria Clara Aderne dos Santos ${ }^{1}$, Sérgio Gorender ${ }^{1}$ \\ ${ }^{1}$ Departamento de Ciência da Computação \\ Instituto de Matemática e Estatística \\ Programa de Pós-Graduação em Mecatrônica \\ Universidade Federal da Bahia (UFBA) - Salvador, BA - Brasil \\ claraaderne@gmail.com, gorendereufba.br
}

\begin{abstract}
Group communication services are important building blocks for fault-tolerant distributed systems. In such a service, membership protocols manages the composition of the communication group considering dynamic groups with incoming and outgoing processes. Algorithms have been presented to solve membership in synchronous and partially synchronous distributed systems, tolerating failures, and the impossibility of membership in asynchronous systems has been proven. Hybrid distributed system models, with synchronous and asynchronous components, have been studied, being relevant for representing current systems, such as systems based on IoT, integrating, for example, industrial plants through the internet. In this paper we present an algorithm that solves the membership problem in the distributed systems model SPA (Synchronous Partitioned). The proposed algorithm allows the existence of several intersecting groups. In addition to the algorithm, we present formal proofs of its properties and implementation results in a simulated environment.
\end{abstract}

Resumo. Serviços de comunicação em grupo são importantes blocos de construção para sistemas distribuídos tolerantes a falhas. Neste serviço, o membership faz a gestão da composição do grupo de comunicação considerando grupos dinâmicos com processos entrando e saindo. Têm sido apresentados algoritmos para solucionar o membership em sistemas distribuídos síncronos e parcialmente síncronos, tolerando falhas, tendo sido provada a impossibilidade do membership em sistemas assíncronos. Modelos de sistemas distribuídos híbridos, com componentes síncronos e assíncronos, têm sido estudados, sendo relevantes por representar sistemas atuais, tais como sistemas baseados em IoT, integrando, por exemplo, plantas industriais através da internet. Neste artigo apresentamos um algoritmo que resolve o problema de membership no modelo de sistemas distribuídos SPA (Síncrono Particionado). O algoritmo proposto permite a existência de diversos grupos com interseção entre eles. Além do algoritmo, apresentamos provas formais de suas propriedades e resultados de implementação em ambiente simulado.

\section{Introdução}

O Modelo Síncrono Particionado de Sistemas Distribuídos (SPA) foi inicialmente apresentado em [Macêdo and Gorender 2008] e [Macêdo and Gorender 2009], como 
um modelo híbrido para sistemas distribuídos tolerantes a falhas, com componentes síncronos e assíncronos. Neste modelo tanto processos como canais de comunicação podem ser síncronos ou assíncronos, permanecendo assim durante a execução do sistema, enquanto não falham. O modelo SPA pode ser caracterizado como um modelo parcialmente síncrono de sistemas distribuídos, modelos que não são síncronos, mas que evitam o resultado da impossibilidade do consenso tolerando falhas em sistemas assíncronos [Fischer et al. 1985] ao considerar algumas características síncronas no sistema [Chandra and Toueg 1996], [Cristian and Fetzer 1999] e [Veríssimo and Casimiro 2002]. A característica fundamental deste modelo é o fato de que processos e canais de comunicação síncronos são agrupados em partições síncronas. Esta característica permitiu que fossem desenvolvidos algoritmos para um detector de defeitos e para um serviço de consenso. Modelos de sistemas distribuídos híbridos, como o SPA, com componentes síncronos e assíncronos, têm sido estudados, sendo relevantes por representar sistemas atuais, como por exemplo sistemas baseados em IoT, integrando plantas industriais através da internet.

Um dos aspectos importantes deste modelo, conforme discutido nestes artigos, é $\mathrm{o}$ fato de poder ter um detector de defeitos da classe $\mathrm{P}$ (Perfeito) em um sistema que não é síncrono [Macêdo and Gorender 2009]. Este detector é obtido caso o modelo satisfaça a propriedade strong partitioned synchrony, ou seja, todos os processos do sistema façam parte de uma partição síncrona. Ainda assim, não teremos um sistema síncrono, pois existirão canais de comunicação assíncronos entre processos deste sistema. Além disto, foi demonstrado que no modelo SPA é possível ter uma detecção de defeitos que, em qualquer situação, satisfaz a propriedade strong accuracy [Macêdo and Gorender 2012], ou seja, um detector de defeitos que não faz detecções incorretas.

Foi apresentado para o modelo SPA um algoritmo de consenso eficiente [Gorender and Macêdo 2011], utilizando o detector de defeitos apresentado anteriormente, e tolerando falhas. O detector de defeitos e o consenso são importantes mecanismos para prover tolerância a falhas em sistemas distribuídos. Um mecanismo também importante para a construção de sistemas distribuídos tolerantes a falhas é um serviço de comunicação em grupo.

Um serviço de comunicação em grupo faz a gestão da formação de grupos de comunicação entre os processos do sistema e provê a comunicação entre os membros dos grupos com garantias diversas, tolerando falhas. Este serviço é formado por dois componentes complementares: um serviço de membership, responsável pela criação dos grupos de comunicação e pela gestão da composição destes grupos, e um serviço de multicast, o qual fornece primitivas de comunicação em grupo multicast, podendo fornecer uma comunicação confiável, e com diferentes critérios de ordenamento [Cristian 1991, Chockler et al. 2001, Anceaume et al. 1995].

O problema do membership é caracterizado pela necessidade de manutenção da composição de grupos de comunicação, assumindo que processos podem solicitar entrar e sair destes grupos, ou falhar, e neste caso precisam ser excluídos do membership. Para que a comunicação ocorra de forma correta e segura, é preciso que os processos que são membros de um grupo mantenham uma visão consistente de sua composição. Esta gestão da composição dos grupos é efetuada através da manutenção de visões sequenciais (views) desta composição instaladas de forma consistente por todos os processos membros. $\mathrm{O}$ 
membership é considerado um problema de acordo, com características equivalentes ao problema do consenso.

O problema do membership em sistemas de comunicação em grupo tem sido investigado em diversos trabalhos [Cristian 1991], [Chockler et al. 2001], [Schiper 2006], [Macêdo and Freitas 2009]. Foram apresentadas soluções para o membership em sistemas síncronos em [Cristian 1991] e [Park et al. 2016], entre outros. Em sistemas assíncronos foi provada a impossibilidade do membership na ocorrência de falhas [Chandra et al. 1996]. Este resultado é similar ao da impossibilidade do consenso em sistemas assíncronos na presença de falhas [Fischer et al. 1985]. Esta impossibilidade ocorre por ser impossível em um sistema assíncrono diferenciar um processo lento de um que falhou.

Para evitar o resultado da impossibilidade do membership em sistemas assíncronos, têm sido apresentadas soluções para o membership em diferentes modelos de sistemas, como, por exemplo, para o modelo Timed Asynchronous [Fetzer and Cristian 1997]. Outras soluções são apresentadas em [Schiper and Toueg 2006], [Schiper 2006], [Ezhilchelvan et al. 1995], [Keidar et al. 2002], [lin and Hadzilacos 1999], entre outros trabalhos. Foram apresentados serviços de comunicação em grupo para sistemas distribuídos híbridos [Macêdo and Freitas 2009][Macêdo et al. 2011], tais como o Sistema Híbrido e Dinâmico [Gorender et al. 2007].

Neste artigo, apresentamos um protocolo de membership para o sistema Síncrono Particionado com a propriedade Strong Partitioned Synchrony. Por assumirmos a propriedade Strong, o algoritmo utiliza o detector de defeito da classe P [Macêdo and Gorender 2009], além do algoritmo de consenso [Gorender and Macêdo 2011], ambos desenvolvidos para o modelo SPA. Este serviço de membership permite a interseção entre grupos de comunicação, com processos podendo ser membros de mais de um grupo ao mesmo tempo. A partir das propriedades assumidas pelo modelo de sistema, e pelo detector de defeitos e consenso, o algoritmo de membership satisfaz propriedades de safety e liveness, garantindo que a visão da composição dos grupos é sempre mantida consistente para todos os seus membros e que as operações relacionadas à gestão dos grupos de comunicação, com processos entrando e saindo destes grupos, são sempre realizadas.

Além do algoritmo e sua descrição e das propriedades e suas provas formais, também apresentamos alguns resultados de experimentos obtidos através da implementação do algoritmo de membership em ambiente simulado de sistemas distribuídos [Silva Freitas and de Araújo Macêdo 2014].

O artigo é composto pelas seguintes seções: na seção 2 são apresentados os trabalhos relacionados; o modelo do sistema é apresentado na seção 3; a seção 4 descreve o modelo SPA, juntamente com os serviços de detecção de falhas e consenso; a seção 5 apresenta o algoritmo de membership proposto e as provas formais de suas propriedades são apresentadas na seção 5.1; a seção 6 descreve a implementação do protocolo e os resultados de testes realizados; e a seção 7 apresenta as conclusões do trabalho. 


\section{Trabalhos relacionados}

Em sistemas distribuídos síncronos, existem soluções para o problema do membership como a apresentada em [Cristian 1991]. [Park et al. 2016] apresenta um protocolo de membership para sistemas distribuídos síncronos que executam sobre uma rede cabeada arbitrária, entre outros.

Motivados pelo resultado da impossibilidade do membership em sistemas assíncronos na presença de falhas [Chandra et al. 1996], têm sido apresentadas soluções para o membership desenvolvidas para sistemas distribuídos parcialmente síncronos, tais como [Ezhilchelvan et al. 1995], [Schiper and Toueg 2006], [Fetzer and Cristian 1997], [Keidar et al. 2002], [lin and Hadzilacos 1999], [Lim and Conan 2014] e [Schiper 2006], entre outros. [Chockler et al. 2001] apresenta uma survey de diversos trabalhos sobre comunicação em grupo discutindo enfoques diferentes. [Anceaume et al. 1995] faz uma análise de formalizações propostas para soluções para o membership, suas características e problemas.

Macêdo e Freitas apresentam em [Macêdo and Freitas 2009] e [Macêdo et al. 2011] o Timed Causal Blocks (TimedCB), um serviço de comunicação em grupo para sistemas distribuídos híbridos, auto-gerenciáveis, baseado no conceito dos causal blocks, o qual foi apresentado em [Ezhilchelvan et al. 1995]. Este trabalho assume um modelo híbrido e dinâmico de sistemas distribuídos, formado por canais de comunicação e processos que podem ser síncronos ou assíncronos. Também assume a existência de um oráculo de QoS, além de serviços de detecção de defeitos e consenso, como descritos em [Gorender et al. 2005]. Embora também seja um modelo de sistemas distribuídos híbrido, o modelo apresentado em [Gorender et al. 2005] é diferente do Síncrono Particionado, com características, comportamentos e propriedades diferentes.

$\mathrm{O}$ algoritmo de membership que apresentamos neste artigo foi desenvolvido para o modelo SPA, utilizando um serviço de deteç̧ão de defeitos da classe P, além de um serviço de consenso, conforme desenvolvidos para este modelo. Embora em um sistema que não é síncrono, o algoritmo apresentado permite efetuar de forma consistente a gestão de grupos de comunicação.

\section{Modelo de Sistema}

Assumimos um sistema distribuído composto por um conjunto de processos (П), os quais se comunicam através de troca de mensagens via canais de comunicação $(C)$. Existem canais de comunicação interligando cada 2 processos. Os processos e os canais de comunicação podem ser síncronos ou assíncronos, e este estado não é alterado durante a execução do sistema. Os processos e canais de comunicação formam um grafo completo não direcionado $D S=\{\Pi, C\}$. O modelo assume a existência de um oráculo temporal que realiza o mapeamento de canais e processos para valores síncronos e assíncronos. $\mathrm{O}$ oráculo é definido pela função de $Q o S$ (quality-of-service) que, por sua vez, é definida para os canais e processos no seu momento de criação sem alteração ao longo do tempo. O sistema satisfaz as definições e propriedades do modelo SPA de sistemas distribuídos, conforme descrito na próxima seção.

Assumimos a existência de uma primitiva de comunicação em grupo reliable multicast $($ rmulticast ()$)$, através da qual mensagens são enviadas para todos os processos 
membros de um grupo de forma confiável, ou seja, é garantida a entrega das mensagens. Também assumimos que esta primitiva satisfaz a propriedade Sincronia Virtual (Virtual Synchrony) conforme descrita em [Chockler et al. 2001], ou seja, "se processos $p$ e $q$ instalam uma mesma view $v$ na mesma view anterior $v^{\prime}$, então qualquer mensagem recebida por $p$ em $v^{\prime}$ é também recebida por $q$ em $v^{\prime \prime}$. Desta forma, é garantido que processos membros de um grupo recebem as mesmas mensagens enviadas por rmulticast, nas mesmas views.

\section{Modelo SPA}

Apresentado em [Macêdo and Gorender 2008] e [Macêdo and Gorender 2009], o Modelo Síncrono Particionado de Sistemas Distribuídos (SPA) é um modelo híbrido, possuindo componentes (processos e canais de comunicação) síncronos e assíncronos. Os processos e canais de comunicação formam partições síncronas, as quais são definidas como sub-grafos completos contidos no grafo do sistema $(D S)$ no qual todos os componentes, processos e canais de comunicação são síncronos. Além disto, assume-se que para cada partição síncrona, não existe outro sub-grafo completo do grafo do sistema que contenha a partição síncrona, fora o próprio grafo $D S$.

O modelo satisfaz uma propriedade de particionamento, a qual pode assumir os valores strong partitioned synchrony ou weak partitioned synchrony. A propriedade é assumida como sendo strong quando todos os processos do sistema (pertencentes ao conjunto П) fazem parte de alguma partição síncrona. A propriedade é assumida como sendo weak quando existem processos que são assíncronos, e que não fazem parte de nenhuma partição síncrona.

Todas as partições síncronas possuem ao menos um processo correto, que não falha durante a execução do sistema. Os demais processos podem falhar por parada sem produzir ações futuras. Também é assumido que os canais de comunicação são confiáveis, ou seja, não perdem ou alteram mensagens. A comunicação entre processos de diferentes partições síncronas, ou entre estes e processos assíncronos, se dá através de canais de comunicação assíncronos.

Neste trabalho assumimos que o modelo satisfaz a propriedade strong partitioned synchrony, ou seja, todos os processos são síncronos e pertencem a partições síncronas.

Foi também apresentado em [Macêdo and Gorender 2008] e [Macêdo and Gorender 2009] um detector de defeitos para o modelo SPA, o qual satisfaz as propriedades strong accuracy e strong completeness, pertencendo à classe de detectores $P$ (Perfect), caso o modelo satisfaça a propriedade strong partitioned synchrony. Em [Gorender and Macêdo 2011] foi apresentado um algoritmo de consenso para o modelo SPA, que utiliza o detector de defeitos. Este algoritmo de consenso satisfaz as propriedades de terminação, validade e acordo uniforme. Como assumimos que o modelo satisfaz a propriedade strong partitioned synchrony, e desta forma temos um detector de defeitos da classe $P$, o consenso tolera falhas de até $n-p$ processos, sendo $n$ o número de processos no sistema, e $p$ o número de partições síncronas. Portanto, podem falhar todos os processos menos aqueles que por definição do sistema devem permanecer corretos, um por partição síncrona. 


\section{Membership no SPA}

O protocolo de membership que apresentamos a seguir foi desenvolvido para o modelo SPA com a propriedade Strong Partitioned Synchrony (Particionamento Forte). O algoritmo utiliza os serviços de detecção de defeitos e de consenso desenvolvidos para o modelo SPA, conforme descritos na seção anterior. Este serviço de membership permite a criação e exclusão de novos grupos de comunicação, e a inclusão e exclusão de processos nos grupos existentes. A cada modificação em um grupo é gerada uma nova visão (view) deste grupo, compartilhada por todos os processos membros do grupo. O serviço permite a interseção entre os grupos, com processos podendo fazer parte de mais de um grupo ao mesmo tempo.

Quando um novo grupo é criado, é atribuída uma identificação representada pela variável $g$. Identificações de grupo são assumidas serem únicas. Cada grupo tem sua composição representada por views, as quais recebem identificações únicas em cada grupo. Estas identificações também indicam a sequência entre as views instaladas em cada grupo. Cada view mantém a lista de processos que compõem o grupo naquele instante. Novas views são instaladas para um grupo quando alguma alteração é realizada em sua composição.

No algoritmo temos as seguintes variáveis:

- $g$ - identificação única de um grupo;

- $v_{k g}^{g}$ - composição da view atual do grupo $g$;

- $k g$ - identificação da view atual do grupo $g$;

- $p_{i}$ - processo $i$, identificado como o processo que está executando o algoritmo localmente;

- $p_{j}$ - processo $j$, um outro processo qualquer.

O protocolo está apresentado no Algoritmo 1 e é dividido em oito tarefas numeradas de 0 a 7 . As tarefas 1 a 7 são concorrentes, sendo que a tarefa 7 pode ter diversas execuções concorrentes, uma para cada grupo do qual o processo faz parte. O serviço é executado em módulos distribuídos, um para cada processo do sistema, sendo que todos os processos executam o mesmo algoritmo. A Task 0 é responsável por preparar o funcionamento do serviço, chamando a Task 2 para acessar o detector de defeitos e criando a lista de grupos group-list que irá conter uma referência para os grupos nos quais o processo é um membro.

Algorithm 1 Módulo do serviço de membership para o processo $p_{i}$.

\section{Task 0 - init}

1: detectFaulty

2: new group - list

Task 1 - createGroup()

3: new group $g$ such that $g$ is a unique value

4: insert $g$ in group - list

5: new view $v_{k g}^{g}$ such that $k g=1$

6: $v_{k g}^{g} \leftarrow p_{i}$

7: new tasklist $-g$

8: run instalView(tasklist $-g$ ) concurrently

9: return $g$ and $\left(k g, v_{k g}^{g}\right)$ 
Task 2 - detectFaulty

10: while true do

11: for each group $g$ do

12: $\quad$ for each $p_{j} \in v_{k g}^{g}$ such that $p_{j} \in$ fault do

13: $\quad$ insert $\left\{p_{j}, g\right.$, " $F D$ " $\}$ in tasklist $-g$

14: $\quad$ end for

15: end for

16: end while

Task 3 - joinGroup $(g)$

17: rmulticast(newView $\left(p_{i}, g\right.$, “join"))

Task 4 - leaveGroup $(g)$

18: rmulticast(newView $\left(p_{i}, g\right.$, "leave"))

19: insert $\left\{p_{i}, g\right.$, "leave" $\}$ in tasklist $-g$

Task 5 - installView(tasklist - $g$ )

20: $p i \_i n_{-} g=$ true

21: while $p i_{i} n_{g}$ do

22: $\quad$ wait for tasklist $-g<>$ vazio

23: $\quad$ select the first task $\left\{p_{j}, g\right.$, task $\}$ in the tasklist $-g$

24: $\quad$ if (task $=$ "join") then

25: $\quad\left\{C P, p_{j}\right.$, task $\} \leftarrow$ consensus(idconsensus $(g, k g+1), v_{k g}^{g} \cup\left\{p_{j}\right\}, p_{j}$, task)

26: $\quad$ else

27: $\quad\left\{C P, p_{j}\right.$, task $\} \leftarrow$ consensus(idconsensus $(g, k g+1), v_{k g}^{g}-\left\{p_{j}\right\}, p_{j}$, task $)$

28: $\quad$ end if

29: $\quad$ if $\left(\right.$ task $=$ "leave") and $\left(p_{j}=p_{i}\right)$ then

30: $\quad$ delete $v_{k g}^{g}$

31: $\quad$ delete tasklist

32: $\quad p i_{i} n_{g}=$ false

33: else

34: $\quad k g=k g+1$

35: $\quad v_{k g}^{g}=C P$

36: $\quad$ delete it from tasklist $-g$

37: $\quad$ if (task $=$ "join") then

38: $\quad$ send to $p_{j}$ (joingroup $(C P, g, k$, tasklist $-g)$ )

39: $\quad$ end if

40: end if

41: end while

Task 6 - newView()

42: when rdeliver(new $\left.\operatorname{View}\left(p_{j}, g, t a s k\right)\right)$ do

43: $\quad$ if (task $=$ "join" and $\left.p_{j} \notin v_{k g}^{g}\right)$ or (task="leave" and $\left.p_{j} \in v_{k g}^{g}\right)$ then

44: $\quad$ insert $\left\{p_{j}, g\right.$, task $\}$ in tasklist $-g$

45: end if

46: end when

Task 7 - joingroupmessage ()

47: when rdeliver(joingroup $(C P, g, k$, tasklist $-g))$

48: if $\left(\nexists v_{k g}^{g}\right.$ such that $\left.p_{i} \in v_{k g}^{g}\right)$ then

49: $\quad$ new group $g$ such that $k g=k$ 


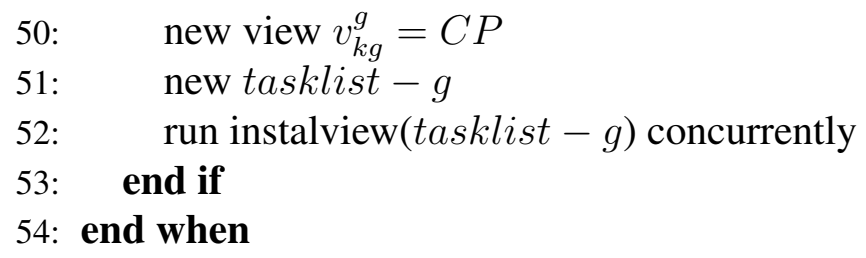

A Task 1 é chamada por um processo quando este deseja criar um novo grupo. A tarefa cria um novo grupo com a identificação única $g$, e com o processo que executa a tarefa $\left(p_{i}\right)$ como membro único em sua primeira view, $v_{k g}^{g}$. A tarefa também cria para o grupo uma lista tasklist-g, para as tarefas pendentes de alteração do grupo, e chama a Task 5 para executar estas alterações, de forma concorrente aos demais grupos dos quais o processo $p_{i}$ faz parte. O novo grupo é inserido na lista group-list. A Task 2 executa o tempo todo de forma concorrente com as demais tarefas do algoritmo e faz uma consulta contínua ao serviço de detecção de defeitos do sistemas. Para cada processo que é detectado como tendo falhado, e que faz parte de algum dos grupos de comunicação no qual o processo $p_{i}$ também faz parte, uma ação para que o processo detectado seja excluído do grupo é inserida na tasklist- $g$ do grupo.

A Task 3 é chamada por um processo $p_{i}$ quando este solicita a sua entrada em um grupo $g$. Esta solicitação é enviada para todos os processos do grupo $g$ através da primitiva rmulticast, para que estes processos instalem uma view com o processo $p_{i}$ como membro. A Task 4 é chamada por um processo $p_{i}$ quando este solicita a sua saída de um grupo $g$ do qual faz parte. A solicitação é enviada por rmulticast para todos os processos do grupo e a ação de saída é inserida na tasklist-g do grupo.

A Task 5 possui várias execuções concorrentes, uma para cada grupo no qual o processo $p_{i}$ faz parte, sendo chamada pela Task 1 ou pela Task 7 . Esta tarefa chama o serviço de consenso com a primeira ação da lista tasklist- $g$ para o grupo $g$ e verifica o resultado do consenso. Se a ação acordada foi a exclusão do próprio processo $p_{i}$, este exclui a tasklist-g e a view do grupo e encerra a execução concorrente desta tarefa para este grupo. Em caso contrário, a nova view acordada no consenso é instalada e a ação de alteração executada é excluída de tasklist-g. Se a ação executada tiver sido um join é enviada uma mensagem com a composição do grupo para o processo $p_{j}$ que solicitou a inclusão.

A Task 6 é executada quando o processo recebe uma mensagem newView solicitando a instalação de nova view por uma ação de entrada de um processo em um grupo (join) ou por uma ação de saída de um processo de um grupo (leave). É feita uma verificação se a solicitação é consistente e a ação é inserida na tasklist-g do grupo. Por fim, a Task 7 é executada por um processo que solicitou sua entrada em um grupo. Quando a inclusão é executada e os processos membros do grupo instalam uma nova view com o processo solicitante como membro do grupo, enviam para este uma mensagem joingroup. Nesta tarefa, o processo que solicitou a inclusão aguarda pelas mensagens joingroup e cria, a partir das mensagens, a view para o grupo, uma tasklist-g, e inicia nova execução concorrente da Task 5.

\subsection{Provas formais de propriedades}

O protocolo de membership apresentado satisfaz propriedades de liveness e safety, conforme definidas em trabalhos clássicos da área, tais como [Chockler et al. 2001]. As pro- 
priedades de liveness possuem o objetivo de garantir que operações solicitadas são realizadas pelo serviço de membership, com a instalação de novas views que representem a execução destas operações. Com estas propriedades provamos que as operações iniciadas são corretamente executadas, efetuando a entrada e saída de processos no membership dos grupos. As propriedades de safety visam provar que em todas as situações as operações sempre geram resultados consistentes, e que não ocorrem estados indesejáveis. Apresentamos primeiro as propriedades de safety e depois as de liveness, além das provas formais mais importantes, quando o espaço permitir.

\subsubsection{Propriedades de safety}

Apresentamos nos teoremas a seguir as propriedades Integridade (self-inclusion), Acordo Uniforme e Validade.

Teorema 5.1.1 (Integridade (self-inclusion)). Se um processo $p$ instala a view $v(g)$ então $p \in v(g)$.

Demonstração. A prova é desenvolvida por contradição, assumindo que o processo $p$ instala a view $v(g)$, do grupo $g$, e que $p$ não pertence a esta view, ou seja, $p \notin v(g)$. Existem duas possibilidades para $p$ instalar a view $v(g)$ : 1) $p_{i}$ já é membro da grupo $g$, tendo instalado a view anterior, e executa Task 5 - installView para o grupo $g$, instalando a view $v(g)$ ou 2) $p_{i}$ não faz parte do grupo, mas solicitou join executando a Task 3, e depois de o join ter sido efetuado pelos membros do grupo, $p$ executa a Task 7, na qual instala a view $v(g)$.

1. $p_{i}$ executa as linhas 34 e 35 da Task 5 - installView para o grupo $g$ - para que esta tarefa esteja em execução pelo processo $p_{i}$ para o grupo $g$, e as linhas 34 e 35 sejam executadas instalando a nova view do grupo, é preciso que $p_{i}$ faça parte do grupo e de seu membership, e neste caso Task 5 já está em execução, e que a condição do comando if da linha 29 dê um resultado falso, e para tal, ou a alteração executada no membership não foi um leave, ou o processo excluído não foi o processo $p_{i} \mathrm{em}$ execução, portanto, neste caso, $p_{i}$ já faz parte do grupo, e como não executou um leave, não foi retirado de seu membership.

2. $p_{i}$ executa as linhas 49 e 50 do algoritmo, na Task 7, instalando o grupo $g$ e a nova view deste grupo - $p_{i}$ executa a Task 7 ao receber uma mensagem joingroup de algum outro processo, $p_{j}$, sendo que um processo $p_{j}$ envia uma mensagem joingroup para um processo $p_{i}$ ao executar a linha 38 na Task 5 do algoritmo; esta linha só é executada se a ação executada foi um join, sendo a mensagem enviada para o processo que foi inserido no membership, e a composição deste membership enviada na mensagem foi o resultado do consenso chamado na linha 25 , e que passa a ter o novo processo, neste caso $p_{i}$, em sua composição, portanto, $p_{i}$ faz parte da nova view instalada.

Como em ambos os casos nos quais um processo $p_{i}$ instala uma view para um grupo, este processo faz parte do membership do grupo, contradizendo a suposição inicial da prova, e provando o teorema.

Teorema 5.1.2 (Acordo uniforme). Processos corretos pertencentes a um mesmo grupo $g$ instalam a mesma sequência de views $v_{i}(g), v_{i+1}(g), \ldots, v_{n}(g)$, a partir do momento em que se juntam ao grupo. 
Demonstração. A prova é realizada por indução sobre o índice $i$ de uma view $v_{i}(g)$.

Passo básico: se dois processos corretos $p_{x}$ e $p_{y}$ são membros de um grupo $g \mathrm{e}$ instalam a view $v_{1}(g)$, e não saem do grupo, irão instalar a view $v_{2}(g)$, posterior à view $v_{1}(g)$.

Assumindo $p_{x}$ e $p_{y}$ membros do grupo $g$, o processo $p_{x}$ instala a view $v_{1}(g)$ executando a Task 5 linhas 34 a 36, e que o processo $p_{y}$ entrou no grupo $g$ após executar um join processado nesta mesma view, a qual é instalada pelo processo ao executar a Task 7 , linhas 49 a 52. Como os processos $p_{x}$ e $p_{y}$ são corretos e não solicitam sair do grupo, ambos executam a Task 5, chamam o consenso da linha 25 ou da linha 27, e executam as linhas 34 a 36 do algoritmo, instalando a próxima view $v_{2}(g)$ (segundo a linha 34 $k g=k g+1$, sendo $k g$ o número de identificação da view).

Passo da indução: se dois processos corretos $p_{x}$ e $p_{y}$ são membros do grupo $g$ e instalam uma view $v_{i}(g)$, e não saem do grupo, $p_{x}$ e $p_{y}$ irão instalar a view $v_{i+1}(g)$, imediatamente posterior à view $v_{i}(g)$.

Assumimos que os processos $p_{x}$ e $p_{y}$ são membros do grupo $g$ e instalaram a view $v_{i}(g)$. Provamos que, como os processos são corretos e não solicitam sair do grupo, ambos irão instalar a view $v_{i+1}(g)$, posterior à view $v_{i}(g)$. Logo após instalar a view $v_{i}(g)$, executando a Task 5, linhas 21 a 41, os processos continuam a execução desta tarefa, na próxima iteração do while da linha 21, uma vez que nenhum dos dois processos saiu do grupo $g$. Os processos irão consultar a próxima operação de suas listas tasklist-g (como o serviço de comunicação é confiável e a primitiva multicast satisfaz a propriedade Sincronia Virtual, todas as mensagens de join e leave chegam aos processos membros do grupo, sendo inseridas em suas tasklist-g, embora podendo ser em ordens diferentes), e chamar o consenso na linha 25 ou 27. Como o consenso satisfaz a propriedade de terminação, os processos terminam a execução do consenso decidindo pelo mesmo valor para instalação da view. Após o consenso, como os processos não solicitam sair do grupo $g$, executam as linhas 34 a 38 do algoritmo, instalando a próxima view do grupo, de numeração $k g=k g+1$ (linha 34), e sendo $k g=i$, a próxima view será igual a $i+1$.

Assim, para qualquer índice $i$ de uma view $v_{i}(g)$, dois processos corretos, membros de um grupo $g$ e que permanecem no grupo, $p_{x}$ e $p_{y}$, a próxima view instalada será sempre de índice $i+1$.

Teorema 5.1.3 (Validade). Toda operação executada pelo serviço de membership é requisitada por algum processo e é executada apenas uma vez.

Devido à falta de espaço deixamos esta prova para uma outra publicação.

\subsection{Propriedades de liveness}

Apresentamos nos teoremas a seguir as propriedades de Terminação e Não-trivialidade. A propriedade Terminação aqui apresentada é similar à propriedade Execução das Operações, conforme encontrada em alguns trabalhos.

Teorema 5.2.1 (Terminação). Se um processo correto p executa joinGroup para um grupo $g$, então, em algum momento caso permaneça correto e g existindo, $p$ se torna membro do grupo g e instala uma view $v(g)$ deste grupo (o mesmo vale para leavegroup). 
Demonstração. Esta prova será desenvolvida por contradição assumindo que um processo correto $p$ execute joingroup (g) (Task 3), solicitando ser membro do grupo $g$, que o grupo $g$ permaneça ativo, e que o processo $p$ nunca instale uma view $v(g)$ deste grupo, passando a ser membro de $g$.

Ao executar a Task 3, joinGroup $(g), p$ envia uma mensagem newView $\left(p_{i}, g\right.$, "join") por multicast para todos os processos membros do grupo $g$ (linha 17). Como a comunicação é confiável, todos estes processos irão receber esta mensagem executando a task 6 - newView com os processos membros de $g$ inserindo a ação $p, g$, "join" em sua tasklist- $g$ (linhas 42 a 46). Quando esta ação for a primeira da tasklist- $g$ de algum processo (linha 23), esse passará a chamar o consenso (linha 25), propondo esta ação. Como todos os processos membros do grupo $g$ possuem esta ação em suas tasklist-g, no pior caso, quando esta entrada for a primeira em todas as tasklist- $g$, o consenso decidirá por ela, e a view gerada terá o processo $p$ como membro, sendo igual à composição da view anterior mais o processo $p$. Como a ação executada foi um join, todos os processos que executaram o consenso executam a linha 38, enviando uma mensagem joingroup $(C P, g, k g$, tasklist-g) com a sua tasklist-g. Ao receber todas estas mensagens joingroup $(C P, g, k g$, tasklist-g), executando a Task 7 - joingroupmessage, $p$ irá instalar a nova view deste grupo, executando as linhas 47 a 54. Em consequência, em algum momento, $p$ irá fazer parte do grupo $g$, instalando uma view deste grupo, o que contradiz a suposição inicial.

A prova com relação á operação leavegroup não será apresentada por falta de espaço.

Teorema 5.2.2 (Não-trivialidade). Se um processo $p$ se junta a um grupo g e ele é, ou se torna, indefinidamente alcançável por um processo $q \neq p$, sendo que q já pertence ao grupo $g$, então, a partir de algum momento $t$, $p$ estará para sempre nas views $v_{i}(g)$ que $q$ instalar.

Devido à falta de espaço esta prova não será apresentada aqui.

\section{Implementação e Resultados}

Foi desenvolvida uma implementação do protocolo de membership em ambiente simulado utilizando o framework HDDSS (Hybrid and Dynamic Distributed System Simulator) [Silva Freitas and de Araújo Macêdo 2014]. Foram implementados no simulador os algoritmos do detector de defeitos e de consenso, conforme descritos para o modelo SPA, e o algoritmo de membership apresentado neste artigo.

O ambiente de simulação foi configurado com canais de comunicação confiáveis, sem perdas de mensagens, e um comportamento determinístico para os canais síncronos, e probabilístico para os canais assíncronos. Todos os processos são síncronos, satisfazendo a propriedade Strong Partitioned Synchyrony. Os experimentos foram executados com um único grupo de comunicação, para a validação do funcionamento do protocolo. Foram utilizados dois cenários, ambos com duas partições síncronas. No primeiro cenário utilizamos 2 processos por partição, totalizando 4 processos e no segundo cenário, utilizamos 4 processos por partição, totalizando 8 processos.

Em cada experimento realizado, após a criação do grupo, são solicitadas operações de joingroup e de leavegroup, às vezes em sequência, às vezes ao mesmo tempo. A eficiência avaliada para o protocolo foi calculada em termos do tempo para a execução de 
cada operação, desde a sua solicitação até a instalação de uma nova view pelos processos do grupo com a operação realizada. No caso de operação gerada pela detecção de um processo (detectFaulty), calculamos o tempo a partir da detecção da falha por um primeiro processo no grupo, até a instalação da view. Para cada cenário fizemos 50 repetições da execução da simulação e, a partir dos dados destas execuções chegamos aos valores apresentados nas tabelas 1 e 2 . O tempo no simulador é calculado em termos de unidades de tempo do relógio interno do framework.

\section{Tabela 1. Dados de média e desvio padrão da eficiência do processamento das operações para o cenário $A$}

\begin{tabular}{|l|l|l|c|}
\hline \multicolumn{4}{|c|}{ Cenário A } \\
\hline $\begin{array}{l}\text { Média Eficiência } \\
\text { Join (4 proces- } \\
\text { sos) }\end{array}$ & 30,16 & $\begin{array}{l}\text { Desvio Padrão } \\
\text { Eficiência Join (4 } \\
\text { processos) }\end{array}$ & 9,78 \\
\hline $\begin{array}{l}\text { Média Eficiência } \\
\text { leave (4 proces- } \\
\text { sos) }\end{array}$ & 26,84 & $\begin{array}{l}\text { Desvio Padrão } \\
\text { Eficiência leave } \\
\text { (4 processos) }\end{array}$ & 21,48 \\
\hline $\begin{array}{l}\text { Média Eficiência } \\
\text { faulty (4 proces- } \\
\text { sos) }\end{array}$ & 16,54 & $\begin{array}{l}\text { Desvio Padrão } \\
\text { Eficiência faulty } \\
\text { (4 processos) }\end{array}$ & \\
\hline
\end{tabular}

Tabela 2. Dados de média e desvio padrão da eficiência do processamento das operações para o cenário $B$

\begin{tabular}{|l|l|l|l|}
\hline \multicolumn{4}{|c|}{ Cenário B } \\
\hline $\begin{array}{l}\text { Média Eficiência } \\
\text { Join (8 proces- } \\
\text { sos) }\end{array}$ & $\begin{array}{l}\text { Desvio Padrão } \\
\text { Eficiência Join (8 } \\
\text { processos) }\end{array}$ & \\
\hline $\begin{array}{l}\text { Média Eficiência } \\
\text { leave (8 proces- }\end{array}$ & 44,80 & $\begin{array}{l}\text { Desvio Padrão } \\
\text { Eficiência leave } \\
\text { sos) }\end{array}$ & $\begin{array}{l}\text { (8 processos) } \\
\text { Média Eficiência } \\
\text { faulty (8 proces- } \\
\text { sos) }\end{array}$ \\
\hline
\end{tabular}

Avaliamos que quanto maior o número de processos em um grupo, maiores os tempos de processamento para cada operação, devido, principalmente ao tempo de troca de mensagens no consenso para estabelecimento da composição da nova view. A variação no número de processos no grupo, à medida em que processos entram e saem, gerou uma variação grande nos tempos para execução das operações, implicando em um desvio padrão alto. O número de processos impacta principalmente na execução do consenso, devido ao alto número de mensagens sendo trocadas, gerando maiores tempos para a transmissão das mensagens nos canais assíncronos.

Os resultados apresentados são preliminares, sendo que ajustes estão sendo realizados no sentido de otimizar a implementação do algoritmo no simulador, e novos experimentos serão realizados para próximos trabalhos, com maior número de processos, e a criação de diversos grupos em diferentes cenários. 


\section{Conclusões}

Neste artigo apresentamos um protocolo de membership para o modelo Síncrono Particionado de sistemas distribuídos, com a propriedade Strong Partitioned Synchrony sendo satisfeita, ou seja, todos os processos pertencem a partições síncronas. O protocolo utiliza um detector de defeitos da classe $\mathrm{P}$ e um algoritmo de consenso. O protocolo satisfaz propriedades de safety e liveness, sendo que algumas de suas provas formais foram apresentadas.

Embora em um sistema não síncrono, o uso de um detector de defeitos da classe $\mathrm{P}$, além de algoritmo de consenso desenvolvido para executar com o mesmo detector, permitem o desenvolvimento de um serviço de membership simples e consistente.

Em trabalhos futuros serão efetuados novos experimentos, em ambiente simulado, com o objetivo de obter uma mais ampla validação deste algoritmo. O serviço de membership será também estendido para também funcionar no modelo SPA caso a propriedade Weak Partitioned Synchrony seja satisfeita, existindo processos assíncronos, que não são membros de partições síncronas.

\section{Referências}

Anceaume, E., Charron-Bost, B., Minet, P., and Toueg, S. (1995). On the Formal Specification of Group Membership Services. Research Report RR-2695, INRIA. Projet REFLECS.

Chandra, T. D., Hadzilacos, V., Toueg, S., and Charron-Bost, B. (1996). On the impossibility of group membership. In Proceedings of the fifteenth annual ACM symposium on Principles of distributed computing, pages 322-330.

Chandra, T. D. and Toueg, S. (1996). Unreliable failure detectors for reliable distributed systems. Journal of the ACM (JACM), 43(2):225-267.

Chockler, G. V., Keidar, I., and Vitenberg, R. (2001). Group communication specifications: a comprehensive study. ACM Computing Surveys (CSUR), 33(4):427-469.

Cristian, F. (1991). Reaching agreement on processor-group membrship in synchronous distributed systems. Distributed Computing, 4(4):175-187.

Cristian, F. and Fetzer, C. (1999). The timed asynchronous distributed system model. IEEE Transactions on Parallel and Distributed Systems, 10(6):642-657.

Ezhilchelvan, P. D., Macêdo, R. A., and Shrivastava, S. K. (1995). Newtop: a faulttolerant group communication protocol. In Proceedings of 15th International Conference on Distributed Computing Systems, pages 296-306. IEEE.

Fetzer, C. and Cristian, F. (1997). A failure aware membership service. In Proceedings of the 16th Symposium on Reliable Distributed Systems, page 157. IEEE.

Fischer, M. J., Lynch, N. A., and Paterson, M. S. (1985). Impossibility of distributed consensus with one faulty process. Journal of the ACM (JACM), 32(2):374-382.

Gorender, S., Macedo, R., and Raynal, M. (2005). A hybrid and adaptive model for fault-tolerant distributed computing. In 2005 International Conference on Dependable Systems and Networks (DSN'05), pages 412-421. IEEE. 
Gorender, S. and Macêdo, R. J. d. A. (2011). Consenso distribuído eficiente no modelo síncrono particionado. In Anáis do XXIX Simpósio Brasileiro de Redes de Computadores e Sistemas Distribuídos (SBRC), pages 587-600.

Gorender, S., Macedo, R. J. d. A., and Raynal, M. (2007). An adaptive programming model for fault-tolerant distributed computing. IEEE Transactions on Dependable and Secure Computing, 4(1):18-31.

Keidar, I., Sussman, J., Marzullo, K., and Dolev, D. (2002). Moshe: A group membership service for wans. ACM Transactions on Computer Systems, 20.

Lim, L. and Conan, D. (2014). Partitionable group membership for mobile ad hoc networks. Journal of Parallel and Distributed Computing, 74(8):2708-2721.

lin, k. and Hadzilacos, V. (1999). Asynchronous group membership with oracles. In Proceedings of the 13th International Symposium on Distributed Computing, pages 70-93.

Macêdo, R. J. d. A. and Freitas, A. E. S. (2009). A generic group communication approach for hybrid distributed systems. In IFIP International Conference on Distributed Applications and Interoperable Systems, pages 102-115. Springer.

Macêdo, R. J. d. A., Freitas, A. E. S., et al. (2011). A self-manageable group communication protocol for partially synchronous distributed systems. In 2011 5th LatinAmerican Symposium on Dependable Computing, pages 146-155. IEEE.

Macêdo, R. J. d. A. and Gorender, S. (2008). Detectores perfeitos em sistemas distribuídos não síncronos. In Anais do IX Workshop de Teste e Tolerância a Falhas (WTF 2008).

Macêdo, R. J. d. A. and Gorender, S. (2009). Perfect failure detection in the partitioned synchronous distributed system model. In 2009 International Conference on Availability, Reliability and Security, pages 273-280. IEEE.

Macêdo, R. J. d. A. and Gorender, S. (2012). Exploiting partitioned synchrony to implement accurate failure detectors. International Journal of Critical Computer-Based Systems 4, 3(3):168-186.

Park, S., Yoo, S., Kim, Y., Lee, S., and Kim, D. (2016). A message efficient group membership protocol in synchronous distributed systems. In Information Technology: New Generations, pages 1249-1254. Springer.

Schiper, A. (2006). Dynamic group communication. Distributed Computing, 18(5):359374.

Schiper, A. and Toueg, S. (2006). From set membership to group membership: A separation of concerns. IEEE Transactions on Dependable and Secure Computing, 3(1):2-12.

Silva Freitas, A. E. and de Araújo Macêdo, R. J. (2014). A performance evaluation tool for hybrid and dynamic distributed systems. ACM SIGOPS Operating Systems Review, 48(1):11-18.

Veríssimo, P. and Casimiro, A. (2002). The timely computing base model and architecture. IEEE Transactions on Computers, 51(8):916-930. 